\title{
The Construction of Sudden Cosmological Singularities
}

\author{
John D. Barrow ${ }^{1}$, S. Cotsakis ${ }^{2}$ and A. Tsokaros ${ }^{3}$ \\ ${ }^{1}$ DAMTP, Centre for Mathematical Sciences, \\ Cambridge University, Cambridge CB3 0WA, UK \\ ${ }^{2,3}$ DICSE, University of the Aegean, Karlovassi 83200, Samos, Greece
}

\begin{abstract}
Solutions of the Friedmann-Lemaitre cosmological equations of general relativity have been found with finite-time singularities that are everywhere regular, have regular Hubble expansion rate, and obey the strong-energy conditions but possess pressure and acceleration singularities at finite time that are not associated with geodesic incompleteness. We show how these solutions with sudden singularities can be constructed using fractional series methods and find the limiting form of the equation of state on approach to the singularity.
\end{abstract}

PACS 98.80.-k, 04.62.+v

In standard Friedmann-Lemaitre cosmological models a fluid is placed in a homogeneous and isotropic spatial geometry whose dynamics is then determined by two independent Einstein equations for three unknown time-dependent functions, the Friedmann metric scale factor, $a(t)$, the fluid density, $\rho(t)$, and fluid pressure, $P(t)$, respectively (with units chosen with $c=8 \pi G=1$ ):

$$
\begin{aligned}
\frac{\dot{a}^{2}+k}{a^{2}} & =\frac{\rho}{3} \\
-2 \frac{\ddot{a}}{a}-\frac{\dot{a}^{2}+k}{a^{2}} & =P .
\end{aligned}
$$

If an equation of state $P=f(\rho)$ is chosen, the system closes and the two remaining unknown functions are determined. Physically reasonable equations of state, like those for perfect fluids, produce well behaved expansion factors with behaviours that offer simple Newtonian interpretations. However, the challenge of providing a compelling explanation for the observed acceleration of the universe in terms of a 'dark energy' fluid has led to an exploration of other less familiar equations of state [1], motivated by the form of bulk viscous stresses [2], situations were $d P / d \rho$ is not everywhere continuous, or scenarios in which there is no equation of state at all. These introduce quite different possibilities into the solution space of Friedmann universes and can produce unexpected types of finite-time singularity which are far softer than the curvature singularities studied previously [3] in connection with geodesic incompleteness [4, 5].

Barrow [6, 7] identified a whole new class of pressure-driven singularities that keep the scale factor, $a$, expansion rate, $\dot{a} / a$, and the density, $\rho$, finite while the pressure, $P$, blows up at a finite time despite the energy conditions $\rho>0$ and $\rho+3 P>0$ holding. Their status as stable solutions of the classical Einstein equations in the presence of small scalar, vector and tensor perturbations has been studied in a gauge covariant formalism [8], and they have also been found to be stable against quantum particle production processes [9]. They have been investigated in a number of different cosmological scenarios and their behaviour has been classified in the light of other types of finite-time singularity that can arise in isotropic and anisotropic cosmologies [10 14]. The formal definitions of these singularities has been discussed by Lake [15] in the case where infinities occur in second derivatives of $a(t)$, but similar examples exist where the sudden singularity occurs in higher derivatives of $a(t)$ and no energy conditions are threatened [7, 10]. Here, we shall provide an alternative systematic way of constructing them.

A sudden singularity will be said to arise everywhere at comoving proper time $t_{s}$ in a Friedmann universe expanding with scale factor $a(t)$ if

$$
\lim _{t \rightarrow t_{s}} a(t)=a_{s} \neq 0, \quad \lim _{t \rightarrow t_{s}} \dot{a}(t)=\dot{a}_{s}<\infty, \quad \lim _{t \rightarrow t_{s}} \ddot{a}(t)=\infty,
$$

for some $t_{s}>0$. From the last requirement we have that, for all constant $M>0$, there exists a constant $\epsilon>0$ such that for $\left|t-t_{s}\right|<\epsilon$ and $t<t_{s}$, we have

$$
\ddot{a}(t)>M \text {. }
$$

By integrating the differential inequality (3), we have

$$
0<\int_{t}^{t_{s}} M d t<\int_{t}^{t_{s}} \ddot{a} d t=\dot{a}_{s}-\dot{a}(t)
$$


and with a further integration we see that for every positive number $M$ we can find a sufficiently small left neighbourhood of $t_{s}$ such that for every $t$ in that neighborhood the scale factor satisfies

$$
a(t)>a_{s}-\dot{a}_{s}\left(t_{s}-t\right)+\frac{M}{2}\left(t_{s}-t\right)^{2} .
$$

For this condition to be satisfied in the neighborhood of $t_{s}$, we must have

$$
a(t)=a_{s}-\dot{a}_{s}\left(t_{s}-t\right)+C\left(t_{s}-t\right)^{n}+\cdots
$$

where $C$ is a constant that depends on $M$, and necessarily we have $1<n<2$.

If $n$ is a rational, the expansion factor can be written as a Puiseux series of the form

$$
a(t)=\sum_{i=0}^{\infty} a_{i}\left(t_{s}-t\right)^{i / s}
$$

where $s>0$ is a natural number.

We can study these kinds of singularities by the dominant balance method [16], wherein a dynamical system $\dot{\mathbf{x}}=\mathbf{f}(\mathbf{x})$ is split into dominant and subdominant terms $\mathbf{f}(\mathbf{x})=\mathbf{f}^{(\mathbf{0})}(\mathbf{x})+\mathbf{f}^{(\mathbf{s u b})}(\mathbf{x})$ and then we search for solutions of the dominant dynamical system $\dot{\mathbf{x}}=\mathbf{f}^{(\mathbf{0})}(\mathbf{x})$ in the form of a Puiseux or $\Psi$ series. In our case, setting $x=a, y=\dot{a}$ we get from (2)

$$
\dot{x}=y, \quad \dot{y}=-\frac{k+y^{2}+x^{2} P}{2 x},
$$

and we require a dominant solution $x=a_{s}$ and $y=\dot{a}_{s}$ both finite, with pressure being infinite. Clearly, these two conditions cannot be simultaneously satisfied.

The root of the problem is that the dominant balance method looks for a singularity of the variable

$$
\mathbf{x}=\left(\alpha_{1}\left(t_{s}-t\right)^{p_{1}}, \alpha_{2}\left(t_{s}-t\right)^{p_{2}}, \cdots\right)
$$

(i.e. at least one of the $p_{i}<0$ ) but we want $\mathbf{x}$ to be finite and $\dot{\mathbf{x}}$ (and so $\ddot{a}$ ) to have a singularity since the pressure term appears in the higher derivative $(\ddot{a})$ equation (2). Despite this structure, the general form of the pressure which leads to this singularity can be found by looking for solutions of (5) of the form

$$
x(t)=\sum_{i=0}^{\infty} c_{1 i}\left(t_{s}-t\right)^{i / s}, \quad y(t)=\sum_{i=0}^{\infty} c_{2 i}\left(t_{s}-t\right)^{i / s},
$$

with $s>1$. We want

$$
c_{10}=\alpha \neq 0, \quad c_{20}=\beta \neq 0,
$$

to hold. In this case, the pressure takes the form

$$
P=\left(t_{s}-t\right)^{h} \sum_{i=0}^{\infty} p_{i}\left(t_{s}-t\right)^{i / s}
$$

and for $P$ to blow up at $t_{s}$, we must have $h<0$. The recursion relations for the $c_{i j}$ are

$$
c_{1 s}=0, \quad 0<i<s, \quad c_{2 i}=-\frac{s+i}{s} c_{1(s+i)}
$$

and

$$
\begin{aligned}
& -\frac{2(s+1) \alpha}{s^{2}} c_{1(s+1)}\left(t_{s}-t\right)^{-(s-1) / s}-\frac{4(s+2) \alpha}{s^{2}} c_{1(s+2)}\left(t_{s}-t\right)^{-(s-2) / s}-\cdots \\
& -\frac{2(s-1)(2 s-1) \alpha}{s^{2}} c_{1(2 s-1)}\left(t_{s}-t\right)^{-1 / s}-\left(4 \alpha c_{1(2 s)}+\beta^{2}+k\right)+ \\
& +\frac{2(s+1)}{s^{2}}\left[\beta(s+1) c_{1(s+1)}-\alpha(2 s+1) c_{1(2 s+1)}\right]\left(t_{s}-t\right)^{1 / s}+ \\
& +\left[\frac{2(s+2)^{2} \beta}{s^{2}} c_{1(s+2)}-\frac{(s+1)(s+3)}{s^{2}} c_{1(s+1)}^{2}-\frac{4(s+1)(s+2) \alpha}{s^{2}} c_{1(2 s+2)}\right]\left(t_{s}-t\right)^{2 / s} \\
& +\cdots=\alpha^{2} p_{0}\left(t_{s}-t\right)^{h}+\alpha^{2} p_{1}\left(t_{s}-t\right)^{h+1 / s}+\alpha^{2} p_{2}\left(t_{s}-t\right)^{h+2 / s}+\cdots
\end{aligned}
$$


By balancing the terms in this equation we can determine the series expansion of the pressure that will lead to a specific sudden singularity. For example, if $n=3 / 2$ in [6]

$$
a(t)=\left(\frac{t}{t_{s}}\right)^{q}\left(a_{s}-1\right)+1-\left(1-\frac{t}{t_{s}}\right)^{n}
$$

we get for the pressure:

$$
P=\frac{3}{2 \alpha t_{s}^{3 / 2}}\left(t_{s}-t\right)^{-1 / 2}-\frac{4 \alpha \alpha_{2}+\beta^{2}+k}{\alpha^{2}}-\frac{9 \beta}{2 \alpha^{2} t_{s}^{3 / 2}}\left(t_{s}-t\right)^{1 / 2}+\cdots,
$$

with

$$
\alpha=\alpha_{s}, \quad a_{i}=\left(a_{s}-1\right)\left(\begin{array}{l}
q \\
i
\end{array}\right)\left(-\frac{1}{t_{s}}\right)^{i}, \quad \beta=-a_{1},
$$

and the density has the form

$$
\rho=\rho_{s}+\frac{9 \beta}{\alpha^{2} t_{s}^{3 / 2}}\left(t_{s}-t\right)^{1 / 2}+\cdots, \quad \rho_{s}=\frac{3\left(k+\beta^{2}\right)}{\alpha^{2}} .
$$

We note here that the asymptotic form of the equation of state has the simple form

$$
\lim _{t \rightarrow t_{s}}\left(P\left(\rho-\rho_{s}\right)\right)=\frac{27 \beta}{2 \alpha^{3} t_{s}^{3}}
$$

and so the fluid behaves like a Chaplygin gas on approach to the sudden singularity. Hence, we see that these formal methods for the analysis of finite-time singularities in dynamical systems allow us to construct cosmological singularities of the sudden type.

In conclusion, we have shown how to construct evolutionary behaviours meeting the conditions for a sudden cosmological singularity using analytic techniques with fractional power series employed to study finite-time singularities in differential equations.

[1] J.D. Barrow, Phys. Lett. B 235, 40 (1990).

[2] J.D. Barrow, Phys. Lett. B 180, 335 (1987); J.D. Barrow, Nucl. Phys. B 310, 743 (1988); J.D. Barrow, in The Formation and Evolution of Cosmic Strings, eds. G. Gibbons, S.W. Hawking \& T. Vaschaspati, CUP, Cambridge (1990), pp. 449-464.

[3] F.J. Tipler, Phys. Lett. A 64, 8 (1977); A. Królak, Class. Quantum Grav. 3, 267 (1986).

[4] L. Fernandez-Jambrina and R. Lazkoz, Phys. Rev. D 70, 121503(R) (2004); L. Fernandez-Jambrina and R. Lazkoz, Phys. Rev. D 74, 064030 (2006); A. Balcerzak and M.P. Dąbrowski, Phys. Rev. D 73, 101301 (2006).

[5] S.W. Hawking and G.F.R. Ellis, The large scale structure of space-time, Cambridge UP, Cambridge (1973).

[6] J.D. Barrow, Class. Quantum Grav. 21, L79 (2004); J.D. Barrow, G.J. Galloway and F.J. Tipler, Mon. Not. Roy. astr. Soc., 223, 835 (1986).

[7] J.D. Barrow, Class. Quantum Grav. 21, 5619 (2004).

[8] J.D. Barrow and S.Z.W Lip, Phys. Rev. D 80, 043518 (2009).

[9] J.D. Barrow, A.B. Batista, J.C. Fabris and S. Houndjo, Phys. Rev. D 78, 123508 (2008)

[10] J.D. Barrow and C.G. Tsagas, Class. Quantum Grav. 22, 1563 (2005)

[11] H. Stefancic, Phys. Rev. D 71, 084024 (2005); E.J. Copeland, M. Sami, and S. Tsujikawa, hep-th/0603057; S. Cotsakis and I. Klaoudatou, J. Geom. Phys. 57 (2007) 1303-1312.

[12] Y. Shtanov and V. Sahni, Class. Quantum Grav. 19, L101, (2002); M.P. Da̧browski, Phys. Rev. D 71, 103505 (2005); S. Nojiri, and S.D. Odintsov, arXiv:hep-th/0412030v1.

[13] S. Nojiri, S.D. Odintsov and S. Tsujikawa, Phys. Rev. D 71, 063004 (2005); C. Cattoen and M. Visser, Class. Quantum Grav. 22, 4913 (2005), M. Dąbrowski, Phys. Lett. B 625, 184 (2005).

[14] M.P. Dąbrowski, T. Denkiewicz and M.A. Hendry, arXiv:gr-qc/0704.1383.

[15] K. Lake, Class Quantum Grav. 21, L129 (2004).

[16] S. Cotsakis, J.D. Barrow, J. Phys. Conf. Ser. 68, 012004 (2007). 